Research Article

\title{
Track-Before-Detect Procedures in AM Radio-Based Passive Radar
}

\author{
Yang Li $\mathbb{D},{ }^{1}$ Hong $\mathrm{Ma}^{2}$ Yuntao Wu $\mathbb{D},{ }^{1}$ and Li Cheng $\mathbb{D}^{1}$ \\ ${ }^{1}$ School of Electrical and Information Engineering, School of Computer Science and Engineering, Wuhan Institute of Technology, \\ Wuhan 430073, China \\ ${ }^{2}$ School of Electric Information and Communications, Huazhong University of Science and Technology, Wuhan 430074, China
}

Correspondence should be addressed to Li Cheng; chengli8102@163.com

Received 30 April 2021; Revised 3 August 2021; Accepted 23 October 2021; Published 13 November 2021

Academic Editor: Hervé Aubert

Copyright (c) 2021 Yang Li et al. This is an open access article distributed under the Creative Commons Attribution License, which permits unrestricted use, distribution, and reproduction in any medium, provided the original work is properly cited.

\begin{abstract}
In amplitude modulation radio-based passive radar, the track-before-detect (TBD) procedures are performed to process long time observation data. This work mainly focuses on the tracking of the time-Doppler and time-azimuth traces of multiple target under real scenarios such as low signal-to-noise ratio, hybrid clutter, severe breaking points, and intersecting traces. A new original approach to deal with the TBD problem of this work is developed. Two types of linear equations according to the simplified radio wave propagation model are formulated, which is the key point of the proposed method. We make the theoretical analysis about how the linear equations can be used to track multiple target, and how multiple target's constant velocities and initial positions can be estimated, which is an additional parameter estimation capability of the proposed method. Both the simulated data and real experimental data are performed with the proposed method and some conventional TBD methods. Several comparisons of the results are given to verify the effectiveness of the proposed method.
\end{abstract}

\section{Introduction}

Passive bistatic radar (PBR) detects and tracks targets by using noncooperative illuminators of opportunity [1]. Compared with other frequency bands, the PBR works in high frequency band and has the advantages of large area coverage, over-the-horizon detection, better stealth target detection, etc. [2-4]. The shortwave (3 30 MHz) amplitude modulation (AM) radio stations are distributed worldwide, transmitted power (typical value is $50 \mathrm{MW}$ ) is far larger than other illuminators of opportunity [1], and the AM radio signal can bounce from the ionosphere and be heard many thousands of kilometers away. This allows the AM radio signal to be received almost at any place throughout the world. However, low bandwidth and time-varying properties limit its detection performance when used in PBR $[2,5]$.

It is a challenge to estimate the time-delay for the bandwidth limitation of AM radio signal; hence, estimating the direction-of-arrival (DOA) of scattered wave is crucial to the AM radio-based PBR [6]. In our previous research, we developed a weak signal enhancement approach [7] and proposed a range-Doppler domain array signal processing (RD-ASP) method [8,9], which can estimate the DOA of scattered wave with high resolution and suppress the clutter simultaneously. Due to the severe time-varying properties of ionospheric propagation environment [10], long time observation is required; thus, using the track-before-detect (TBD) technology is a great choice to detect targets.

This work mainly focuses on the tracking of the timeDoppler and time-azimuth traces for targets in sight; however, the propagation environment, including the sea, land, and ionospheric propagation [10-12], is extremely complicated, which leads to a challenging problem due to unknown and varying number of multitarget, model mismatch, lower signal-to-noise ratio (SNR), hybrid clutter, severe breaking points, intersecting traces, etc. Typical TBD strategies include Kalman filter (KF) [13, 14], Hough transform (HT) [15-17], velocity filtering (VF) [18-21], particle filtering (PF) [22-25], dynamic programming (DP) [26-30], and Greedy algorithm [31]. Their basic concept, 
possible advantages, and limitation for application related to this work are summarized as follows:

(1) KF-TBD: In earlier passive radar applications [13], the $\mathrm{KF}$ is used to track and associate the timeDoppler and time-DOA traces of multiple target; it is the closest one to the application scenario of this paper. However, the tracking cycle contains many steps that require human intervention, and it is difficult to be applied flexibly for the categories of traces are complicated [14].

(2) HT-TBD: The HT transforms the problem of track detection in data space into the problem of peak detection in parameter space [15]. The weak target is detected by noncoherent integration of multiframe measurement data. It has the advantage of not discarding data in a time history, while previous data is discarded in most conventional TBD methods. It is tolerant of large gaps in the data and does not require uniformly spaced data samples [16]. Additionally, it can perform target detection, data association, track initiation, and track maintenance at the same time [17]. The limitation of HT-TBD is that only the trace with straight line or specific curve forms can be detected [32], and the performance can be greatly deteriorated if the target movement is not in accordance with the assumed measurement model.

(3) VF-TBD: The VF is a $3 \mathrm{D}$ matched filter [18], it assumes a constant target velocity, and the signature of the target remains constant over time. Both the velocity information and the target position can be estimated [19]. Since the exact target velocity is unknown, a velocity filter bank is used to cover the possible target velocities, and the space should be close enough to achieve a specified maximum SNR loss due to mismatch. This approach could result in a large number of filters required; hence, it will increase the computational complexity [20]. Besides, it cannot separate some targets with very close velocities; such scenario appears in the following experimental data. Furthermore, the measurements of VF-TBD are generally the velocity or location $[19,21]$, which limits its application.

(4) PF-TBD: The PF is a simulation-based Monte Carlo method. The main idea is to represent the required posterior density function by a set of random samples with associated weights and to compute estimates based on these samples and weights [22]. It is flexible to handle the nonlinear and/or nonGaussian models. However, both the Markov transition matrix and target state space model need to be predetermined; if either one deviates from the true values greatly, the detection and tracking performance will decrease dramatically [23]. Additionally, this approach suffers from the curse of dimensionality as the number of targets increases [24, 25, 28].
Hence, it does not result in a generally applicable algorithm.

(5) DP-TBD: The DP algorithm is probably the most widely used method. It integrates the measurements along possible target traces, returning as declared targets for which the measurement sum exceeds a threshold. It performs the equivalent of an exhaustive search of all possible target traces $[26,27]$. A drawback of DP-TBD is that the computational complexity and memory resource requirements are potentially high, because it involves processing a high-dimensional data, processing by multiframe detection, and batch/sliding window processing. Furthermore, the problems about the initial values for tracking, the threshold determination, and the prior knowledge of the number of targets are also difficult for application [31].

(6) G-TBD: The Greedy algorithm (GA) is much simpler and more rapid than DP for solving some optimal solutions. It makes a locally optimal choice at each stage and then obtains a global optimal or suboptimal solution. It can achieve the same or similar performance with DP under some restrictions [31]. However, to achieve a good performance, the threshold determination is very complex, and the performance will decrease dramatically if the trace is composed of large gaps.

In this paper, a novel TBD method is developed. Firstly, due to the facts that the distance between the transmitter and target is far larger than the distance between the receiver and target, and the flight level of target is generally invariant, we made a simplified radio wave propagation model to approximate the complex ionospheric propagation model. Then, based on this simplified model, a very simple constant velocity linear equation, which includes the target measurement parameters, azimuth angle, and Doppler frequency, is formulated. Theory indicates that the constant velocity equation can be used to perform target detection, data association, track initiation, and track maintenance at the same time, which is very similar to the HT-TBD. Then, another simple initial position equation is formulated to separate multiple target with the same or very close velocities in exceptional circumstances. Finally, the traces of interest can be tracked through backtracking with the detected points of multiple target.

The main contribution of this paper includes the following: (1) to the best of our knowledge, this work represents a new original approach to deal with the TBD problem in particular application. It does not belong to any existing category. (2) The proposed method not only can handle the problem of tracking the traces of interest, but also has the ability of estimate the velocities and initial positions at the same time, which would be beneficial for flight trajectory tracking in further processing. (3) The theory, simulation, and real data experiment show the simplicity, easy implementation, and good performance of the proposed method. 
The rest of the paper is organized as follows: the basic signal model and conventional techniques are provided in Section 2. The conventional techniques are described in Section 3. The proposed method and its implementation are developed in Section 4. Some simulation examples are presented in Section 5. Experimental results are shown in Section 6. A brief conclusion appears in Section 7.

\section{System Description}

2.1. The Passive OTHR. The experimental AM radio-based PBR system $[9,33]$ is equipped with a uniform circular array (UCA), which consists of 16 monopole antennas with $10 \mathrm{~m}$ length for each, and the array's diameter is $38 \mathrm{~m}$. After received by the wide-band digital channelized receiver, several strong signals in the AM radio band are downconverted to zero intermediate frequency. After the calibration, array signal is formed for further processing (Figure 1).

In the current research, the signal and data processing generally include the following 6 procedures:

(1) DOA Estimation and Beamforming [34, 35]: Estimating the DOA of direct-path, and obtaining the direct-path signal as reference signal through beamforming technology.

(2) Direct Signal Cancellation [36, 37]: Suppressing the direct-path component in each channel of the array signal to obtain the direct-path-free array signal.

(3) Coherent Processing [3, 9, 38]: Calculating the crossambiguity functions of the reference signal and direct-path-free array signal, then constructing the virtual array signal in which the scattered signals are significantly enhanced.

(4) Scattered Wave DOA estimation [8, 9]: estimating the DOAs as well as the Doppler shifts of scattered signals to obtain the azimuth-Doppler maps. The clutter suppressing procedure is included in this stage.

(5) TBD Processing [14]: Tracking and associating the time-azimuth-Doppler traces on the azimuthDoppler maps.

(6) Flight Trajectory Tracking [13]: Using the timeazimuth-Doppler data to track the target's flight trajectory with advanced tracking method.

2.2. Doppler and Azimuth Traces. In one field experiment, a set of real data with 118 seconds length is collected. The AM radio transmitter station is located more than $1500 \mathrm{~km}$ away from the receiver. Radio signal's frequency is $15.105 \mathrm{MHz}$, and the azimuth DOA of direct path is about $44^{\circ}$. The coherent processing time equals $4 \mathrm{~s}$ for each frame, and there are 115 frames in all, which indicates that there exists a $3 \mathrm{~s}$ overlap between every two frames (sliding window processing). In the following, we use the superscript $(\cdot)^{t}$ to represent the frame sequence (equivalent to time sequence), $t=0,1, \ldots, 115$.
The Automatic Dependent Surveillance-Broadcast (ADS-B) information is received and recorded with the AirNav RadarBox when collecting the data. Figure 2 displays the flight trajectories of 8 civil aircraft targets with ADS-B information.

By performing with the RD-ASP method [4], 115 twodimensional DOA-Doppler maps are obtained. Because the UCA's elevation resolution is low, the elevation angle is fixed to $75^{\circ}$ in RD-ASP; only the azimuth angle scans from $1^{\circ}$ to $360^{\circ}$ with $1^{\circ}$ interval. Figure 3 shows an azimuth-Doppler map at $t=80$. The Doppler bins are $-32 \sim 32 \mathrm{~Hz}$, and the angle bins are $1 \sim 360^{\circ}$. Several (azimuth, Doppler)-pair peaks can be distinguished clearly on the map.

Then, the following three steps are performed: firstly, detecting the peaks with empiric threshold (or constant false alarm rate detection) and only retaining the peaks' values and positions; secondly, extracting the maximum value along the azimuth angles $1 \sim 360^{\circ}$ at each Doppler bin to construct the one-dimensional Doppler values for all the 115 time sequences, and then stacking them to form a two-dimensional time-Doppler map, which is shown in Figure 4; thirdly, extracting the maximum value along the Doppler bins $-32 \sim 32 \mathrm{~Hz}$ at each azimuth angle to construct the one-dimensional azimuth values for all the 115 time sequences, and then stacking them to form a two-dimensional time-azimuth map, which is shown in Figure 5. It is noticed that the peaks in Figures 4 and 5 can be associated with the peaks' (azimuth, Doppler)-pair in Figure 3.

In Figures 4 and 5, it can be clearly seen that hundreds of peaks form continuous or intermittent traces, and some obvious traces even intersect. The TBD problem is how to track multiple targets' azimuth and Doppler traces in a continuous long time observation without detecting the targets in advance.

\section{Conventional Techniques}

The DP-TBD and G-TBD are chosen for comparison for they are easy to be applied in this work, and their performances are as good as those of many existing state-of-the-art methods.

3.1. Propagation Model. Assuming the target flight with constant velocity and constant height, so the Doppler shift of scattered wave is independent of vertical sense. The radio wave propagation model for target in sight can be simplified to a two-dimensional model as shown in Figure 6.

The relative parameters are stated as follows: $X$ and $Y$ axes are the east and north directions on Earth's surface; $T$ $(\mathrm{Tx}), \mathrm{O}(\mathrm{Rx})$, and $\mathrm{P}$ (Tar) are the position of transmitter, received array, and target, respectively; $p_{x}$ and $p_{y}$ are $P$ (Tar) projections along $X$ and $Y$ axes; $v$ is the velocity of a target; $v_{x}$ and $v_{y}$ are $v$ projections along $X$ and $Y$ axes; $\varphi_{d}$ and $\varphi_{s}$ are the DOA of direct path and scattered wave. The angles, $\varphi_{d}$ and $\varphi_{s}$, are clockwise starting from $Y$ axis. Since the distance between $\mathrm{O}(\mathrm{Rx})$ and $\mathrm{T}(\mathrm{Tx})$ (generally more than $1000 \mathrm{~km}$ ) is much further than the distance between $\mathrm{O}(\mathrm{Rx})$ and $\mathrm{P}$ (Tar) (less than $50 \mathrm{~km}$ ), the DOAs of direct path incidents at $\mathrm{O}$ $(\mathrm{Rx})$ and $\mathrm{P}$ (Tar) can be approximately regarded as the same. 


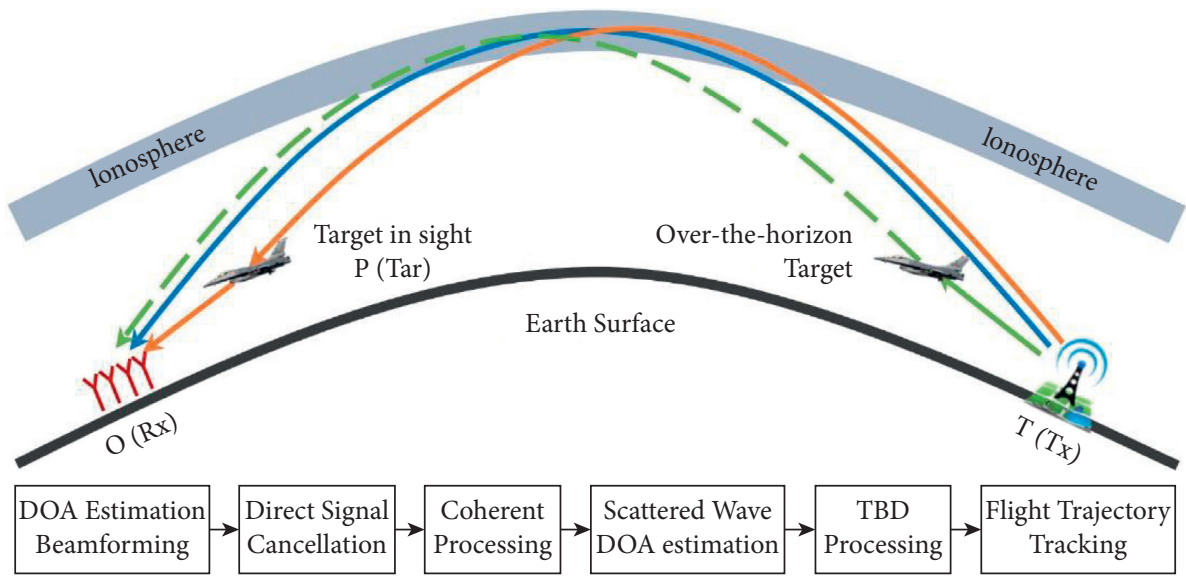

FIGURE 1: Illustration of operational concept and signal processing flowchart for AM radio-based passive radar.

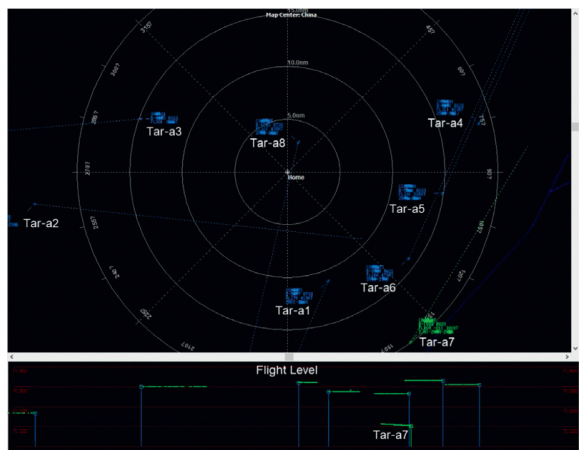

Figure 2: Flight trajectory tracking display in AirNav RadarBox at $t=60$.

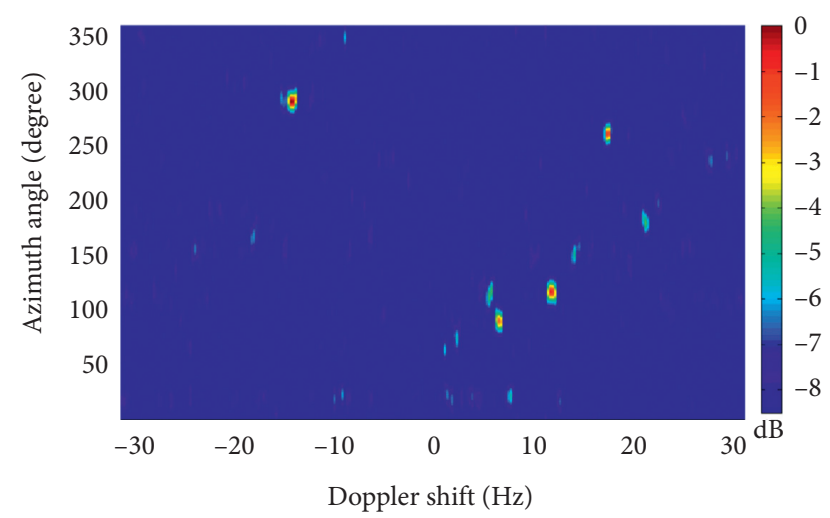

Figure 3: Azimuth-doppler map at $t=80$.

\subsection{Mathematical Model}

3.2.1. Target State Model. The target motion could be modeled in the state space form by

$$
\left\{\begin{array}{l}
p_{x}^{t}=p_{x}^{0}+v_{x} t \\
p_{y}^{t}=p_{y}^{0}+v_{y} t
\end{array}, \quad t=1, \ldots, 115 .\right.
$$

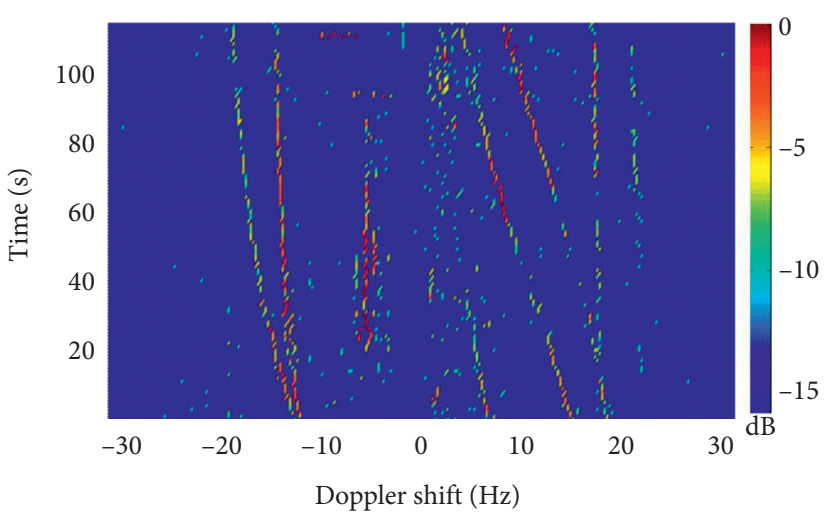

FIgURE 4: Time-doppler map.

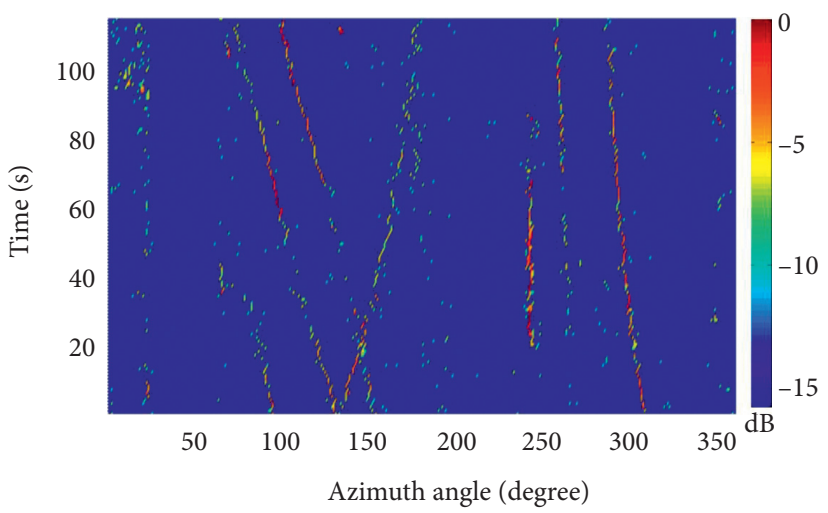

Figure 5: Time-azimuth map.

3.2.2. Target Measurement Model. In the application, the measurements are considered in azimuth-Doppler plane. The target state at the $t$-th frame $\mathbf{x}^{t}$ consists of azimuth $\varphi_{s}^{t}$ and its change rate $\dot{\varphi}^{t-1}$, Doppler frequency $f_{. d_{-1}}^{t}$ and its change rate $\dot{f}_{d}^{t-1}$. It is defined as $\mathbf{x}^{t}=\left[\varphi_{s}^{t}, \dot{\varphi}^{t-1}, f_{d}^{t}, f_{d}^{d-1}\right]^{T}$. The target state evolution can be described by the Markov process

$$
\mathbf{x}^{t} \approx \mathbf{F} \mathbf{x}^{t-1}+\mathbf{n}^{t},
$$




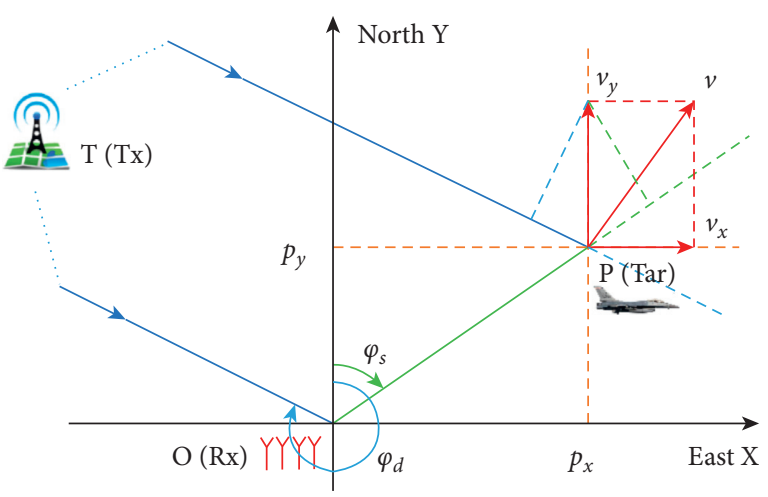

FIgURE 6: Radio wave propagation model.

where $\mathbf{n}$ denotes the noise matrix, $\mathbf{F}=\mathbf{I}_{2} \otimes\left[\begin{array}{cc}1 & \Delta t \\ 0 & 1\end{array}\right], \otimes$ is the Kronecker product, $\mathbf{I}_{2}$ is the two-dimensional identity matrix, and $\Delta t$ is the time between consecutive scans.

The measurement model is suitable for targets moving with slow maneuvers. With regard to the target motion, target usually keeps in the same azimuth/Doppler cell or moves into its neighborhood azimuth/Doppler cells during frame interval, since it is hard for targets to cross several azimuth/Doppler cells during short time interval. Hence, for simplicity, the change rate $\dot{\varphi}^{t-1}$ and $\dot{f}^{t-1}$ can be set equal to zero on condition that the surveillance region covers maximum transfer cells.

3.3. Application of Typical TBD Methods. The surveillance region of adjacent frames consists of a $N_{\varphi} \times N_{f}$ grid of cells, where $N_{\varphi}$ and $N_{f}$ are the number of cells in azimuth angle and Doppler frequency axis, respectively. Therefore, the measurements recorded at frame $t$ are a matrix with size $N_{\varphi} \times N_{f}, i=1, \ldots, N_{\varphi}, j=1, \ldots, N_{f}$

$$
z_{i j}^{t}= \begin{cases}A^{t}+n^{t}, & \text { target in, } \\ n^{t}, & \text { no target in, }\end{cases}
$$

where $z_{i j}^{t}$ denotes the value on the azimuth-Doppler map in the surveillance region cell $(i, j)$ at $t$-th frame.

In measurements preprocessing, the first threshold $V_{\mathrm{LT}}$ is applied to each frame. For $t=1,2, \ldots, N_{t}$, we apply

$$
z_{i j}^{t}= \begin{cases}z_{i j}^{t}, & \text { if } z_{i j}^{t} \geq V_{\mathrm{LT}}, \\ 0, & \text { if } z_{i j}^{t}<V_{\mathrm{LT}} .\end{cases}
$$

The implementation of G-TBD and DP-TBD generally includes the following 4 steps:

(1) Initialization

Setting the initial value at $t=1$ : target state $\mathbf{x}^{1}=\left(\varphi_{s}^{1}, f_{d}^{1}\right)$, and merit function $I\left(\mathbf{x}^{1}\right)=z_{i j}^{1}$.

(2) Recursion

For $t=2,3, \ldots, N_{t}$, recursive calculating the stage merit function

$$
\begin{aligned}
\text { G - TBD: } I\left(\mathbf{x}^{t}\right) & =I\left(\mathbf{x}^{t-1}\right)+\max _{\mathbf{x}^{t}}\left[z_{i j}^{t}\right], \\
\Psi^{t}\left(\mathbf{x}^{t}\right) & =\arg \max _{\mathbf{x}^{t}}\left[z_{i j}^{t}\right], \\
\text { DP - TBD: } I\left(\mathbf{x}^{t}\right) & =\max _{\mathbf{x}^{t-1}}\left[I\left(\mathbf{x}^{t-1}\right)\right]+z_{i j}^{t}, \\
\Psi^{t}\left(\mathbf{x}^{t}\right) & =\arg \max _{\mathbf{x}^{t-1}}\left[I\left(\mathbf{x}^{t-1}\right)\right] .
\end{aligned}
$$

\section{(3) Termination}

Setting the second threshold $V_{T}$, when $t$ reaches the last frame $N_{t}$, a detection result is declared: if $\left\{\hat{x}^{N_{t}}\right\}=\left\{\mathbf{x}^{N_{t}}: I\left(\mathbf{x}^{N_{t}}\right)>V_{T}\right\}$, declare the detection of a target.

(4) Backtracking

For all $\hat{x}^{t}$, for $t=N_{t}-1, \ldots 1, \hat{x}^{t}=\Psi^{t+1}\left(\hat{x}^{t+1}\right)$. The recovered trace estimation is $\left\{\hat{x}^{1}, \hat{x}^{2}, \ldots, \hat{x}^{N_{t}}\right\}$.

\section{Proposed Method}

4.1. Constant Velocity Equation. As shown in Figure 6, at $t$-th time sequence, the velocity's magnitude along the $\mathrm{O} \longrightarrow \mathrm{P}$ path is $\left(v_{x} \sin \varphi_{s}^{t}+v_{y} \cos \varphi_{s}^{t}\right)$, and along the $\mathrm{P} \longrightarrow \mathrm{T}$ path, it is $-\left(v_{x} \sin \varphi_{d}+v_{y} \cos \varphi_{d}\right)$. Hence, the velocity's magnitude along the $\mathrm{O} \longrightarrow \mathrm{P} \longrightarrow \mathrm{T}$ path can be expressed as

$$
v_{O \longrightarrow P \longrightarrow T}=v_{x}\left(\sin \varphi_{s}^{t}-\sin \varphi_{d}\right)+v_{y}\left(\cos \varphi_{s}^{t}-\cos \varphi_{d}\right) \text {. }
$$

Then, the Doppler shift at $t$-th time sequence can be calculated by

$$
f_{d}^{t}=-\frac{1}{\lambda}\left[v_{x}\left(\sin \varphi_{s}^{t}-\sin \varphi_{d}\right)+v_{y}\left(\cos \varphi_{s}^{t}-\cos \varphi_{d}\right)\right]
$$

where $\lambda$ is the wavelength. Expression (2) can be rewritten as

$$
\left(\sin \varphi_{s}^{t}-\sin \varphi_{d}\right) v_{x}+\left(\cos \varphi_{s}^{t}-\cos \varphi_{d}\right) v_{y}+\lambda f_{d}^{t}=0
$$

Since the target's velocities, $v_{x}$ and $v_{y}$, are constant values, we call the expression (8) as "constant velocity equation." If we regard $v_{x}$ and $v_{y}$ as variables, for every $\left(\varphi_{s}^{t}, f_{d}^{t}\right)$ pairs, equation (8) represents a series of straight lines, and the lines corresponding to the same target will inevitably intersect at the same point, which is the target's velocity. Furthermore, with the continuous change of $\left(\varphi_{s}^{t}, f_{d}^{t}\right)$ pairs, the inclination angles of the lines cluster and continuously increase or decrease along with the time sequences. Therefore, targets with different velocities can be separated through equation (8).

The measurement space and parameter space of constant velocity equation are shown in Figure 7, and it is very similar to the data representation of Hough transform [16].

4.2. Initial Position Equation. In case several targets have the same or very close velocities, their constant velocity equation lines will intersect at the same/close point $\left(\widehat{v}_{x}, \widehat{v}_{y}\right)$, which represents their common velocity. To separate these targets, we can use the following relationship: 


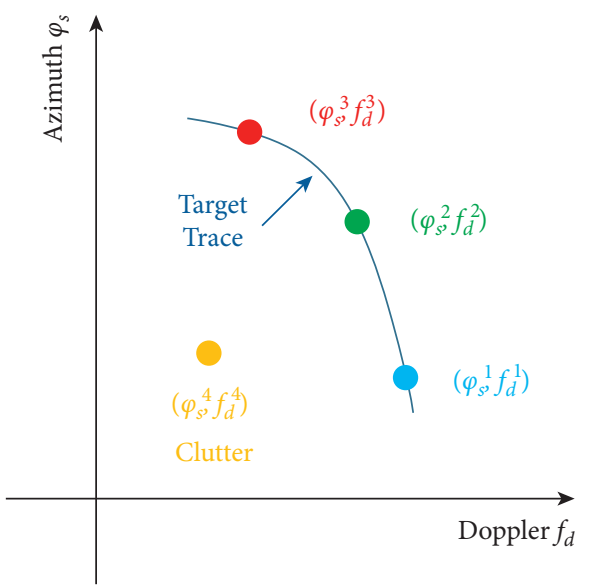

(a)

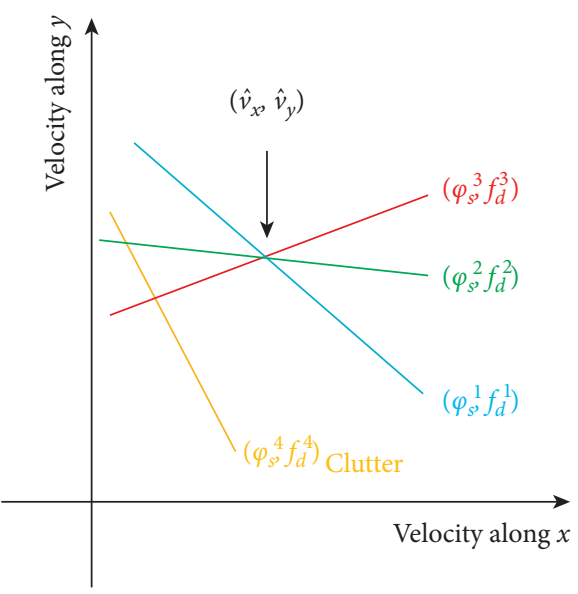

(b)

FIGURE 7: Measurement space and parameter space of constant velocity equation. (a) Measurement data space. (b) Velocity parameter space.

$$
\tan \varphi_{s}^{t}=\frac{p_{x}^{t}}{p_{y}^{t}}=\frac{p_{x}^{0}+\widehat{v}_{x} t}{p_{y}^{0}+\widehat{v}_{y} t} .
$$

Expression (9) can be rewritten as

$$
p_{x}^{0}-\tan \varphi_{s}^{t} p_{y}^{0}+\left(\widehat{v}_{x}-\tan \varphi_{s}^{t} \widehat{v}_{y}\right) t=0
$$

Since the target's initial positions, $p_{x}^{0}$ and $p_{y}^{0}$, are invariable values, we call the expression (10) as "initial position equation." If we regard $p_{x}^{0}$ and $p_{y}^{0}$ as variables, for every $\varphi_{s}^{t}$, equation (10) represents a series of straight lines, and the lines corresponding to the same target will inevitably intersect at the same point, which is the target's initial position. Therefore, targets with the same velocity can be separated through equation (10).

It can be seen that the first constant velocity equation plays a prime role in the proposed method. And the fact that velocity is constant is one of the preconditions for application. Hence, in the following, we call the proposed method as Constant Velocity-TBD (CV-TBD).

4.3. Implementation. The implementation of the proposed $\mathrm{CV}-\mathrm{TBD}$ is summarized as follows:

Step 1: After the measurements preprocessing with threshold $V_{\mathrm{LT}}$, detecting the peaks $\left(\varphi_{s}^{t}, f_{d}^{t}\right)_{k}, k=1,2$, $\ldots$, on all the $t=1,2, \ldots, 115$ azimuth-Doppler maps.

Step 2: Plotting the constant velocity equation map: plotting lines for all $\left(\varphi_{s}^{t}, f_{d}^{t}\right)_{i}$ with equation (8) in range $\left(v_{x}, v_{y}\right) \in[-300,300] \mathrm{m} / \mathrm{s}$ on map-A.

Step 3: Detecting $M$ targets and estimating their velocities: detecting the intersecting lines on map-A, using the positions of intersection points to represent targets' velocities $\left(\widehat{v}_{x}, \widehat{v}_{y}\right)_{m}, m=1,2, \ldots, \mathrm{M}$, and then recording the detected targets' $\left(\varphi_{s}^{t}, f_{d}^{t}\right)_{m}$.

Step 4: Plotting the initial position equation map: plotting lines for all $\left(\widehat{v}_{x}, \widehat{v}_{y}\right)_{m}$ and $\left(\varphi_{s}^{t}\right)_{k}$ with equation (10) in range $\left(p_{x}^{0}, p_{y}^{0}\right) \in[-100,100] \mathrm{km}$ on map-B.
Step 5: Detecting $\mathrm{N}$ targets and estimating their initial positions: detecting the intersecting lines on map-B, using the positions of intersection points to represent targets' initial positions $\left(\widehat{p}_{x}^{0}, \widehat{p}_{y}^{0}\right)_{n}, n=1,2, \ldots, N$, then recording the detected targets $\left(\varphi_{s}^{t}, f_{d}^{t}\right)_{n}$.

Step 6: Plotting the detected points $\left(\varphi_{s}^{t}, f_{d}^{t}\right)_{m \text { or } n}$ in accordance with time sequence to form continuous tracking traces.

\subsection{Further Remarks}

4.4.1. Detection of Intersection Points. The detection of straight lines' intersection points on map-A and map-B can be regarded as the same problem about the detection of sine curves' intersection points in Hough transform; therefore, the accumulator array method [39] is a good choice to be used to detect the intersection points of interest. The problem that the intersection point may extend to a small area because of the measure error, model error, and noise appears in both the CV-TBD and HT-TBD. It is not easy to deal with this problem. Some effective approaches have been developed to meet the requirement $[16,39]$.

4.4.2. Constraint of Intersection Points. The intersecting lines corresponding to an actual target have the following features: (a) they intersect at the same point in map-A, and the corresponding velocity of the intersection point is around $250 \mathrm{~m} / \mathrm{s}$ (for civil aircraft detection); (b) they intersect at the same point in map- $\mathrm{B}$, and the corresponding position of the intersection point is about $5 \sim 50 \mathrm{~km}$ (for short range detection and subject to antenna's pattern coverage); (c) the angles of inclination cluster together and continuously increase or decrease with time sequence.

4.4.3. Special Targets. When several targets have the same or very close velocities but with different initial positions, the lines corresponding to one detected point in Step 3 can form more points in Step 5, leading to $M<N$. When several 
targets have the same or very close initial positions but with different velocities, the lines corresponding to one detected point in Step 5 can form more points in Step 3, leading to $M>N$.

4.4.4. Computational Complexity. The dominant computational complexity of the CV-TBD is determined by the detection of the intersection points. The accumulator array method is recommended to be used. In this case, the dominant computational complexity of the CV-TBD is the same as the complexity of accumulator array method $[39,40]$.

4.4.5. Compared with Other TBD Methods. The property comparison of the CV-TBD, HT-TBD, and DP-TBD/GTBD is summarized in Table 1.

4.4.6. Limitation of the CV-TBD. On one hand, the CV-TBD takes advantage of the particular scenario that the distance between Tx and target is far larger than the distance between $\mathrm{Rx}$ and target, and the flight level of target is invariant. On the other hand, these two model conditions limit its application.

\section{Simulation}

5.1. Simulation Parameters. Simulation parameters are listed as follows: the path length of $\mathrm{T}(\mathrm{Tx})$ to $\mathrm{O}(\mathrm{Rx})$ is $1500 \mathrm{~km}$; signal's frequency is $15 \mathrm{MHz}$; the azimuth DOA of direct path is $45^{\circ}$; there exists a single target with initial position $\left(p_{x}^{0}, p_{y}^{0}\right) \approx(10,-15) \mathrm{km}$, and constant velocity $\left(v_{x}\right.$, $\left.v_{y}\right)=(100,200) \mathrm{m} / \mathrm{s}$; measure frame $t=1, \ldots, 100$. Target amplitude fluctuation is assumed to follow the Swerling models of types 0 [41], the noise are Gaussian-distributed, and the SNR definition on the azimuth-Doppler plane refers to [37]. The time-Doppler trace and the time-azimuth traces with $\mathrm{SNR}=12 \mathrm{~dB}$ are shown in Figure 8 .

The CV-TBD, G-TBD, and DP-TBD are performed with the simulated data. It is difficult to preset the optimum parameters for three methods; we try to put them to the same standard as possible: the preprocessing threshold $V_{\mathrm{LT}}$ equals $4 \mathrm{~dB}$; the resolution of azimuth and Doppler cell is $1^{\circ}$ and $0.5 \mathrm{~Hz}$, respectively, for all; the surveillance region of adjacent frames is \pm 3 cells for G-TBD and DP-TBD; batch window processing is adopted for DP-TBD with window size $N_{t}=5$ [29]. The initial values are manually set equal to actual values for G-TBD and DP-TBD.

5.2. Linear Equation Map. After performing Steps 1 3, the constant velocity equation map is obtained. As shown in Figure 9, we can see that (a) there are many lines; each line corresponds to a peak on all the $t=1, \ldots, 100$ azimuthDoppler maps. (b) Some lines cluster and intersect at the same point, and they can be regarded as detected targets. (c) Detected targets' velocities can be measured at the intersection points $\left(\widehat{v}_{x}, \widehat{v}_{y}\right) \approx(100,200) \mathrm{m} / \mathrm{s}$, which is equal to actual value. (e) Some "disorder" lines do not meet the actual target's features, and we mark them "Unwanted" and discard them.

Then, choosing $\left(\widehat{v}_{x}, \widehat{v}_{y}\right)$ and $\left(\varphi_{s}^{t}\right)$ corresponding to the detected points in Figure 9, the initial position equation map is obtained after performing Steps $4 \sim 5$. Figure 10 shows that (a) all of the lines intersect at the same point. (b) Detected targets' initial positions can be measured at the intersection points on the map $\left(\widehat{p}_{x}^{0}, \widehat{p}_{y}^{0}\right) \approx(10,-15) \mathrm{km}$, which is equal to the actual value.

5.3. Tracked Trace. Performing Step 6 with recorded target' time sequence, azimuth, and Doppler values, we can plot the continuous time-Doppler trace and time-azimuth trace of the detected target. The continuous trace is simply formed through connecting the adjacent detected points using line segments.

Figure 11 shows the tracked time-Doppler trace and time-azimuth trace of three methods, and the results show that (a) not all the points of 100 frames can be detected by CV-TBD, because some points' values are below the preprocessing threshold $V_{\mathrm{LT}}$. (b) There exists a false alarm point, and repeated simulation tests show that the same phenomenon appears occasionally. Fortunately, such false alarm points usually deviate far from actual trace; therefore, they can be found and deleted easily through smooth tracking.

5.4. Detection Probability. In Figure 12, the curves of detection probability $(\mathrm{Pd})$ versus different SNRs are plotted using 1000 trials. Pd-a and Pd-D denote the Pd of azimuth and the Pd of Doppler, respectively. Pd-ap denotes the Pd that is calculated with the number of detected points divided by 100 , while $\mathrm{Pd}$-a denotes the Pd that is calculated with the number of detected points on the tracked trace divided by 100. We take a $3 \times 3$ cell about its expected location and define a detection as any crossing within this cell.

The results in Figure 12 show that (a) the Pd-ap and PdDp of CV-TBD are relatively lower, because some points' values are below the preprocessing threshold $V_{\mathrm{LT}}$ so that they are discarded. (b) The Pd-a and Pd-D of CV-TBD are apparently higher than other Pd. (c) The Pd-a and Pd-D of CV-TBD cannot achieve $100 \%$ even when the SNR is high; it is because there exist occasional false alarm points. (d) The Pd-a equals Pd-D for CV-TBD, whereas the Pd-a is lower than Pd-D for G-TBD and DP-TBD, especially in low SNR case.

\section{Experimental Results}

Steps $1 \sim 6$ are performed to track the traces in Figures 4 and 5 , and also the results calculated by ADS-B information are used for reference (it can be regarded as actual value).

6.1. Constant Velocity Equation Map. After performing Steps $1 \sim 3$, the constant velocity equation map is obtained. As shown in Figure 13, we can evaluate the following (a) There are hundreds of lines, and each line corresponds to a peak on 
TABLe 1: Property comparison of the CV-TBD, HT-TBD, and DP-TBD/G-TBD.

\begin{tabular}{|c|c|c|c|}
\hline Property & CV-TBD & HT-TBD & DP-TBD/G-TBD \\
\hline Motion model & $\begin{array}{r}\text { Velocity is constant, and } \\
\text { enough to tar }\end{array}$ & $\begin{array}{l}\text { The traces to be tracke } \\
\text { straight lines }\end{array}$ & $\begin{array}{c}\text { Allow a certain degree of model } \\
\text { errors }\end{array}$ \\
\hline $\begin{array}{l}\text { Measurement } \\
\text { space }\end{array}$ & Azimuth angle and Doppler frequency & $\begin{array}{c}\text { The measured points lie in a straight } \\
\text { line }\end{array}$ & Points satisfy the motion model \\
\hline Parameter & $\begin{array}{r}\text { Velocity \& position } \\
\text { line }\end{array}$ & $\begin{array}{l}\text { Hough parameter space sinusoids } \\
\text { lines }\end{array}$ & 1 \\
\hline Track initiation & Anto im & Auto im & Add \\
\hline $\mathrm{Da}$ & A & A & Additional $\mathrm{pr}$ \\
\hline $\mathrm{Mu}$ & ry & Not & $\begin{array}{l}\text { Previous data is discarded in multi- } \\
\text { frame processing }\end{array}$ \\
\hline $\begin{array}{l}\text { Dete } \\
\text { decla }\end{array}$ & $\begin{array}{l}\text { Straight lines intersect at a point (by } \\
\text { accumulator) }\end{array}$ & $\begin{array}{r}\text { Sinusoids lin } \\
\text { (by }\end{array}$ & Merit function ex \\
\hline Othe & $\begin{array}{c}\text { The velocity and initial position can be } \\
\text { estimated }\end{array}$ & Some specific curves can be detected & $\begin{array}{c}\text { Slowly manoeuvring targets can be } \\
\text { handled }\end{array}$ \\
\hline
\end{tabular}
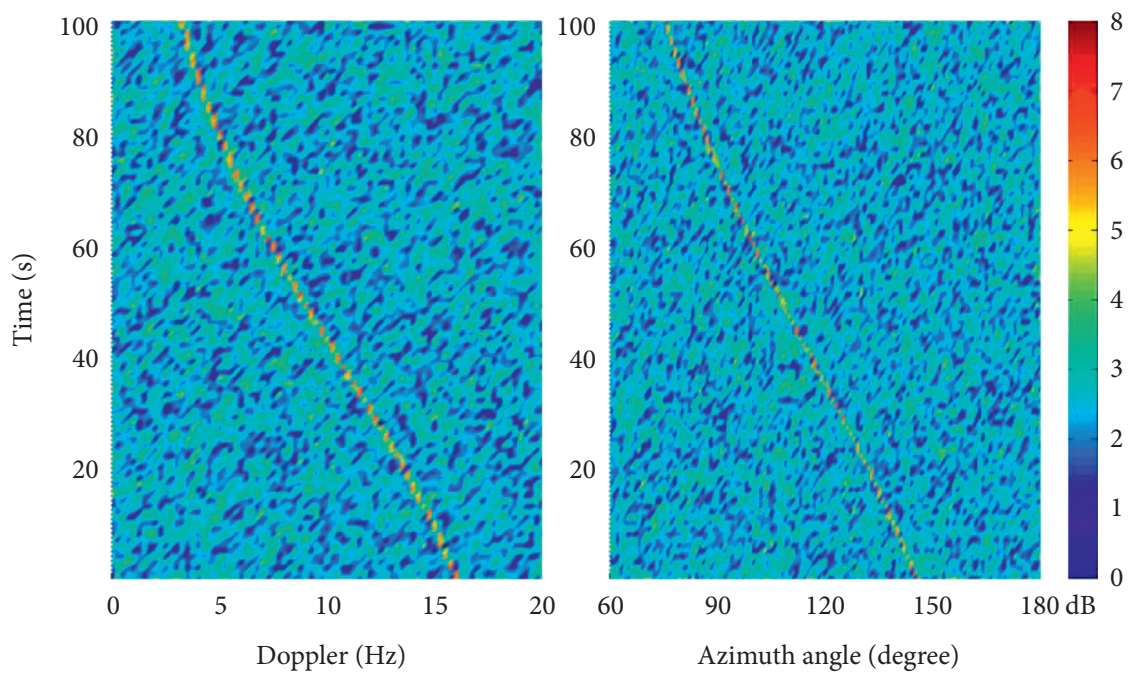

FIgURE 8: The time-doppler and time-azimuth traces of simulation data.

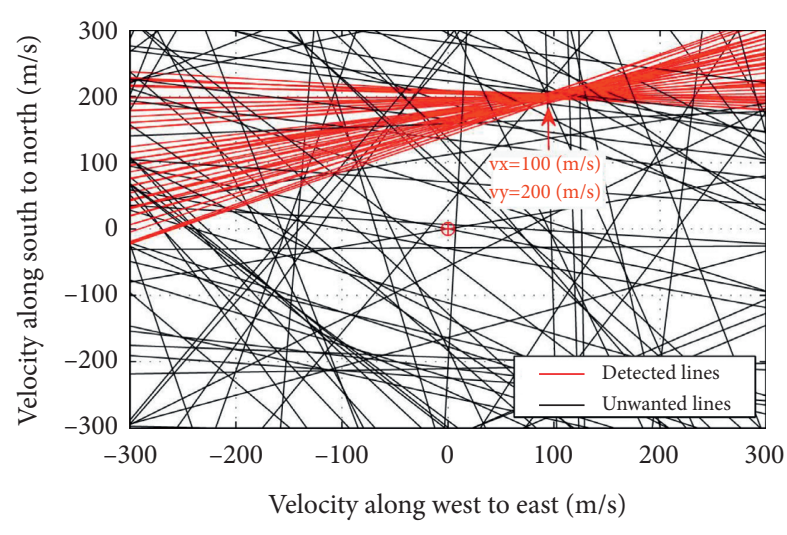

Figure 9: Constant velocity equation map.

the 115 azimuth-Doppler maps. (b) Some lines cluster and intersect at the same point (actually, it extends to a small area); they can be regarded as detected targets, and we mark them with "Tar 1 3." (c) Detected targets' velocities can be measured at the intersection points. (d) Some intersecting

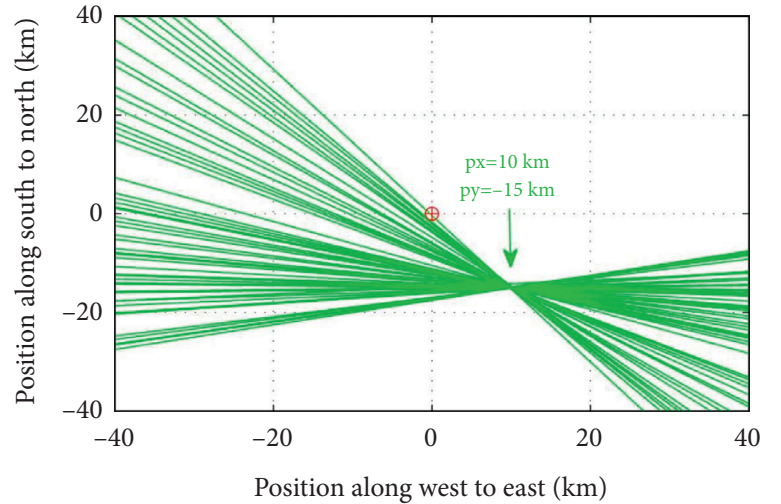

FIGURE 10: Initial position equation map.

lines' inclination angles extend greatly; it is likely that there exist several targets with close speeds, and we mark them as "Uncertain" for the further processing. (e) A great number of lines do not meet the actual target's features, and we mark 

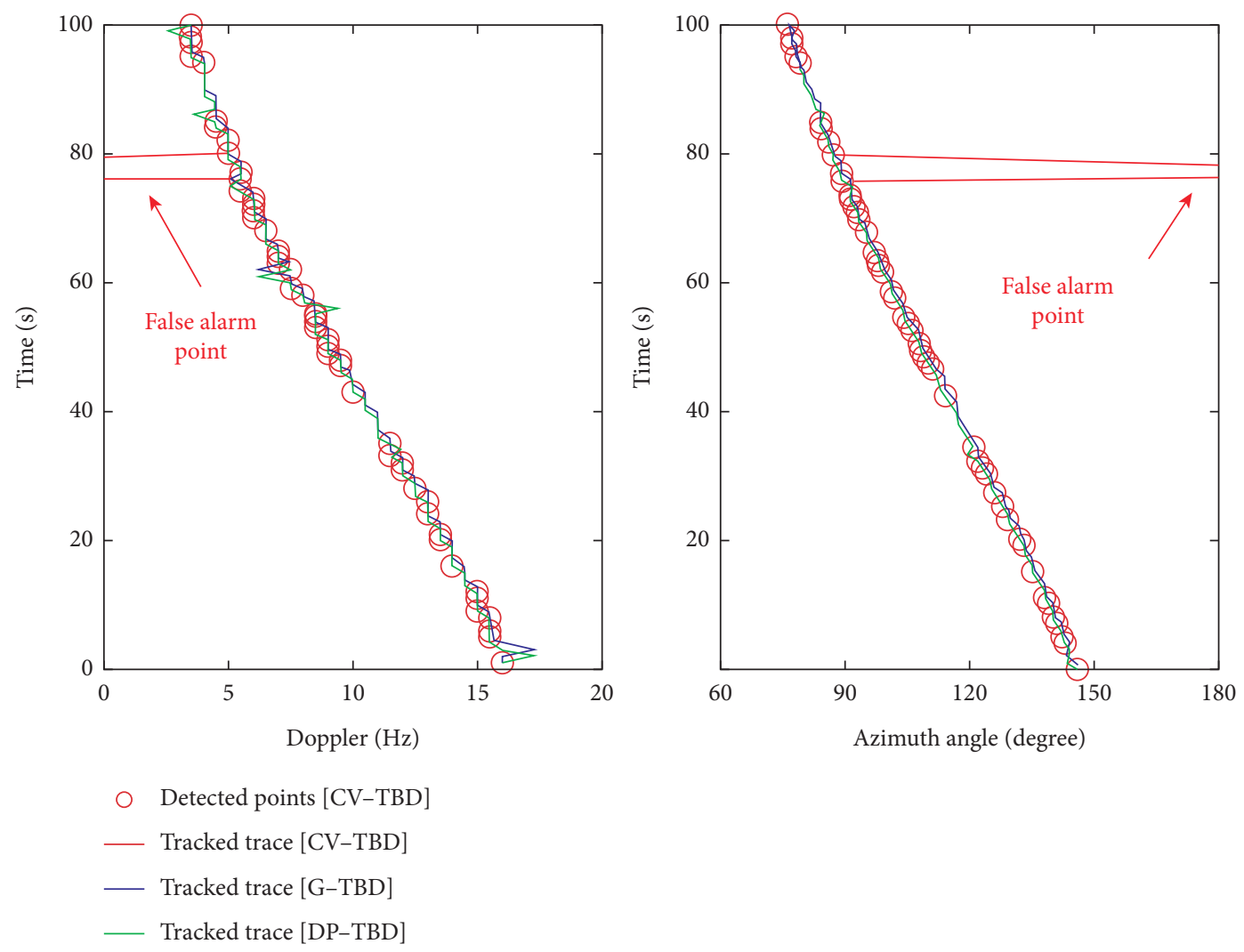

FIgURE 11: Tracked time-doppler trace and time-azimuth trace.

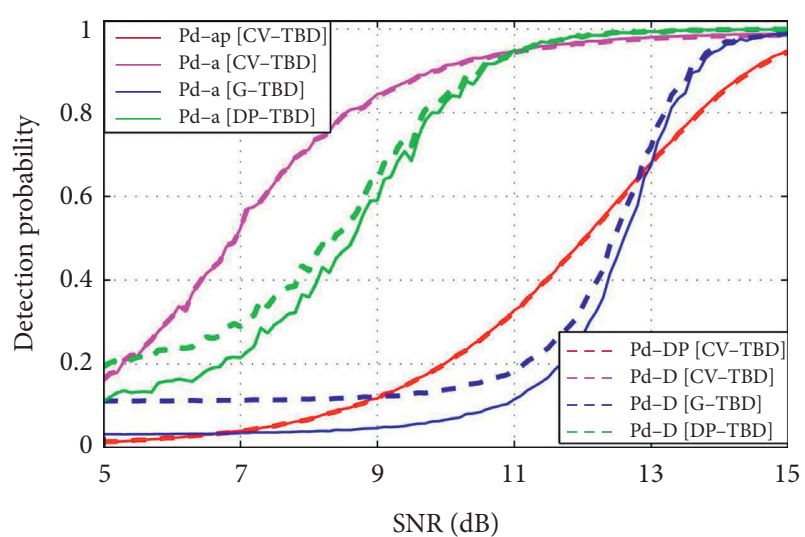

FIGURE 12: Detection probability versus different SNRs.

them as "Unwanted" and discard them. Comparing Figure 13 with Figure 14, we can also find that (f) the positions of intersection points and the shape of corresponding lines in Figure 13 are very similar with the actual results in Figure 14. (g) The "Unwanted" lines in Figure 13 are proved to be clutter. (h) Three close targets, Tar-a4, a5, and a6, are successfully detected but failed to be separated in Figure 13. (i) Tar-a7 and a8 are failed to be detected.

6.2. Initial Position Equation Map. Afterwards, choosing $\left(\widehat{v}_{x}, \widehat{v}_{y}\right)_{m}$ and $\left(\varphi_{s}^{t}\right)_{k}$ corresponding to the detected points in Figure 13, the initial position equation map is obtained after

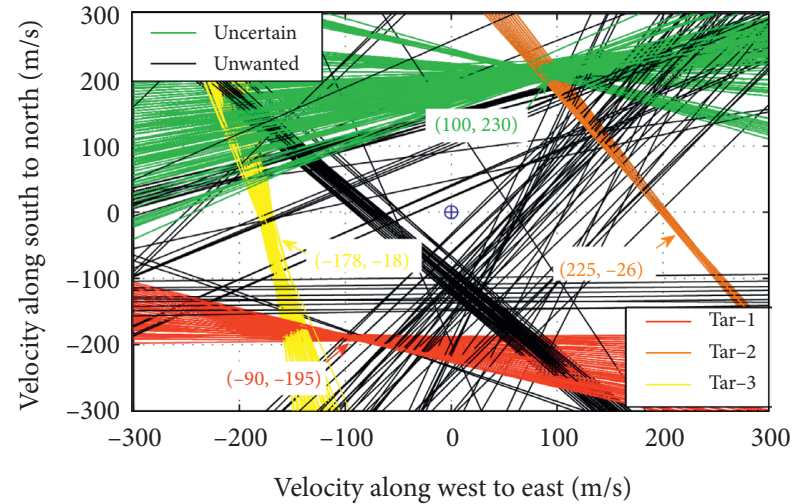

FIgURE 13: Constant velocity equation map with measured data.

performing Steps 4 5. Figure 15 shows that (a) the "Uncertain" lines are mainly intersecting at four different points, and we regard them as detected targets, mark them with "Tar 4 7," and record them. (b) Detected targets' initial positions can be measured at the intersection points on the map. (c) A few lines do not meet the actual target's features, and we mark them as "Unwanted" and discard them. (d) The positions of intersection points and the shape of corresponding lines in Figure 15 are very similar with some actual results in Figure 16.

6.3. Tracked Traces. Finally, performing Step 6 with recorded targets' time sequence, Doppler, and azimuth values, we can plot the continuous 7 time-Doppler traces and 7 time- 


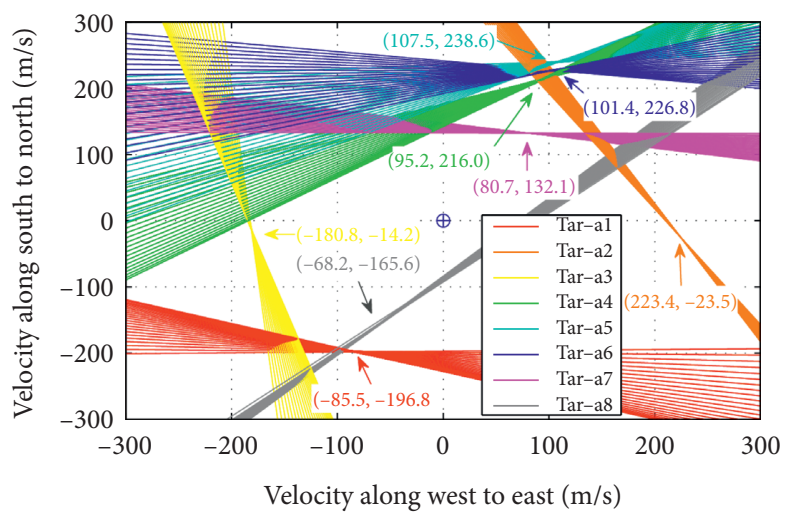

FIgURE 14: Constant velocity equation map with ADS-B information.

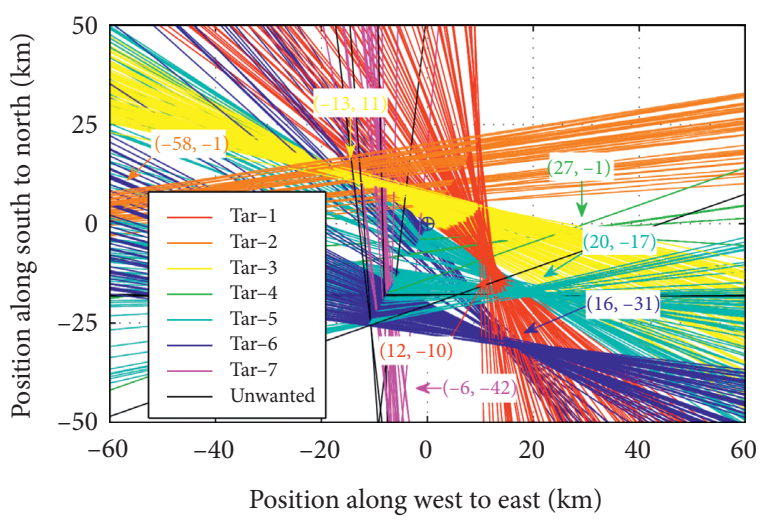

FIGURE 15: Initial position equation map with measured data.

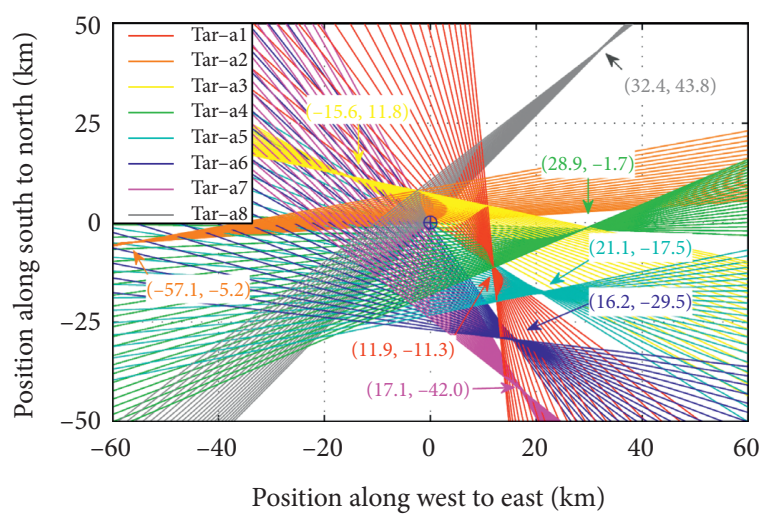

FIGURE 16: Initial position equation map with ADS-B information.

azimuth traces of all the detected targets. The G-TBD and DP-TBD are chosen for comparison. It is difficult to preset the optimum parameters for G-TBD and DP-TBD, some parameters are set with empiric values, and the implementation for G-TBD and DP-TBD is performed with manual intervention appropriately, such as setting the initiation point and removing the redundant and repetitive traces, so as to make the results better.

The results in Figures 17 and 18 show that (a) the CVTBD (color lines), G-TBD, and DP-TBD track at least 6

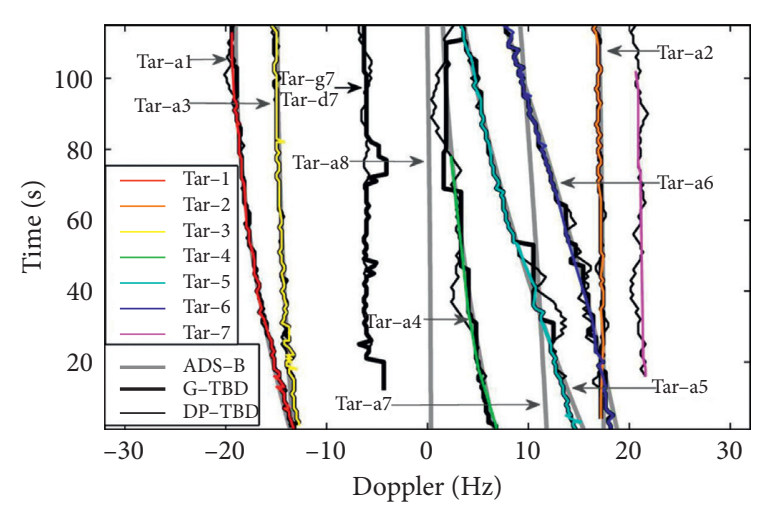

FIgURE 17: TBD result of time-doppler traces.

traces successfully, which are close to the traces in Figures 4 and 5. (b) Three methods are good at tracking the strong traces, that is, the Tar- $(1,2,3)$. (c) Three methods track the intersecting traces successfully, that is, the Tar- $(1,6)$. (d) For some traces with severe breaking points, such as the Tar-(4, 5 ), it is obviously that the performance of the CV-TBD outperforms that of G-TBD and DP-TBD.

Figure 19 gives the root mean square error (RMSE) of 6 targets' traces with 3 TBD methods, and the values calculated with ADS-B information are used for reference. RMSE $=\sqrt{\sum_{t=1}^{N_{r}}\left(y_{e}^{t}-y_{a}^{t}\right)^{2} / N_{r}}$, where $N_{r}$ is the number of detected points for each trace, $y_{e}$ is the estimated value of azimuth or Doppler, and $y_{a}$ is the corresponding value of ADS-B. The reason we calculate the RMSE of azimuth and Doppler independently is that even if only one of them is available, such as the time-Doppler trace, the flight trajectory tracking is realizable under certain conditions [33]. In Figure 19, "a-" denotes the RMSE of azimuth angles, and "D" denotes the RMSE of Doppler frequencies. Results show that the RMSE values of 6 targets calculated by CV-TBD are lower than others in most cases.

Notice that there are some missing detections and false alarms: (a) the flight level of Tar-a7 is too low, and the position of Tar-a8 is almost above the array; hence, they are failed to be detected due to antenna's pattern limitation; (b) Figure 17 shows that the Doppler shift of Tar-a8 is close to 


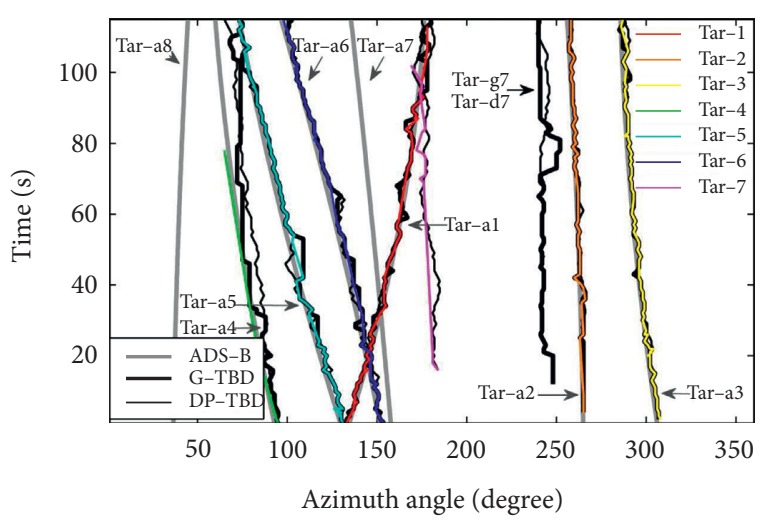

FIgURE 18: TBD result of time-azimuth traces.

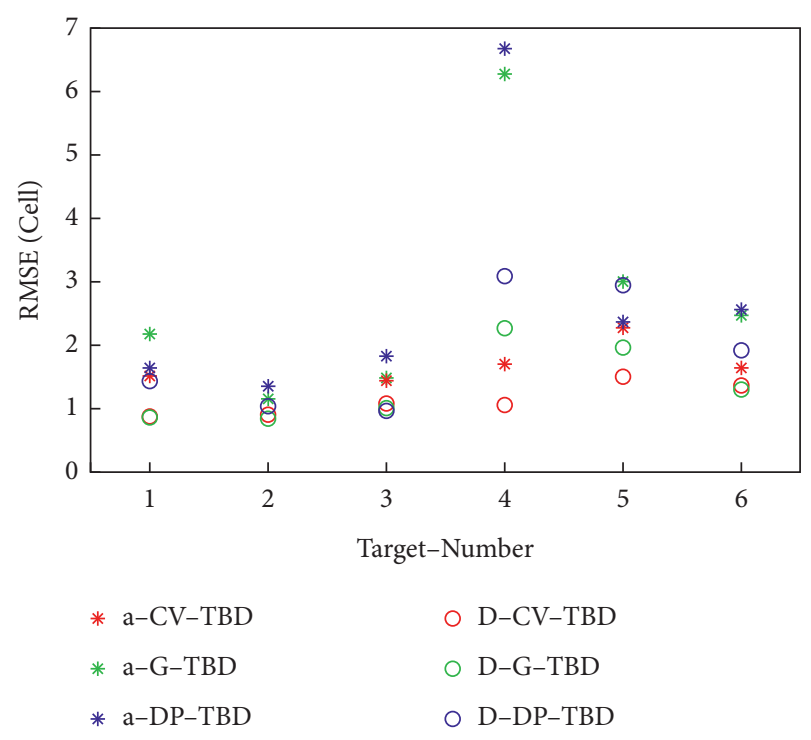

FiguRE 19: RMSE of 6 targets' traces.

$0 \mathrm{~Hz}$; hence, it has been suppressed as direct path in the previous Direct Signal Cancellation procedure; (c) the tar-g7 tracked by G-TBD is a false alarm; (d) the false alarm Tar-7 is not regarded as a normal target for its flight trace is not a straight line after the further flight trajectory tracking processing.

Finally, although the proposed CV-TBD has the ability to roughly estimate the target's constant velocity and initial position, we do not aim to use them to locate and track target directly, but we use them as initial values to track the target's flight trajectory with advanced target tracking method, such as the extended Kalman filter [13].

\section{Conclusion}

We developed a novel TBD method for AM radio-based $\mathrm{PBR}$ application. The proposed method formulates the constant velocity equation and the initial position equation and proves that the lines of linear equation corresponding to an actual target will certainly intersect to the same point, so that they can be tracked. Additionally, the theory indicates that multiple target's constant velocities and initial positions can be estimated with the positions of intersected points on constant velocity equation map and initial position equation map, respectively. Both the simulation and real experimental results show the advantages of the proposed CV-TBD in terms of tracking intersecting traces, breaking point traces, and estimating the constant velocities and initial positions of multiple targets.

\section{Data Availability}

The software code data used to support the findings of this study are currently under embargo, while the research findings are commercialized. Requests for data, 12 months after publication of this article, will be considered by the corresponding author.

\section{Conflicts of Interest}

The authors declare that there are no conflicts of interest regarding the publication of this paper.

\section{Acknowledgments}

This work was supported by the Hubei Provincial Natural Science Foundation of China (Grant No. 2020CFB650), and the National Natural Science Foundation of China (Nos. 61771353 and 62071188).

\section{References}

[1] H. Griffiths and C. Baker, An Introduction to Passive Radar, Artech House, Norwood, MA, USA, 2017.

[2] J. M. Thomas, H. D. Griffiths, and C. J. Baker, "Ambiguity function analysis of digital radio mondiale signals for $\mathrm{HF}$ passive bistatic radar," Electronics Letters, vol. 42, no. 25, pp. 1482-1483, 2006.

[3] G. Fabrizio, F. Colone, P. Lombardo, and A. Farina, "Adaptive beamforming for high-frequency over-the-horizon passive radar," IET Radar, Sonar \& Navigation, vol. 3, no. 4, pp. 384-405, 2009.

[4] G. Fabrizio, A. Zadoyanchuk, D. Francis, and V. Nguyen, "Using emitters of opportunity to enhance track geo-registration in HF over-the-horizon radar," in Proceedings of the 2016 IEEE Radar Conference (RadarConf), Philadelphia, PA, USA, May 2016.

[5] G. Fabrizio, J. Thomolaris, N. Spencer, C. L. Cheng, and B. Ng, "Over-the-horizon aircraft detection using skywave AM-radio broadcast signals," in Proceedings of the 2015 IEEE Radar Conference, Arlington, VA, USA, May 2015.

[6] P. Hu, Q. Bao, and Z. Chen, "DOA estimation method in multipath environment for passive bistatic radar," International Journal of Antennas and Propagation, vol. 2019, Article ID 7419156, 9 pages, 2019.

[7] L. Cheng, Y. Li, L. Zou, and Y. Qin, "DOA estimation for highly correlated and coherent multipath signals with ultralow SNRs," International Journal of Antennas and Propagation, vol. 201910 pages, Article ID 2837315, 2019.

[8] Y. Li, H. Ma, L. Cheng, and D. Yu, "Method for bearing estimation of target for amplitude modulation radio-based passive radar application," Electronics Letters, vol. 54, no. 6, pp. 383-385, 2018. 
[9] Y. Li, H. Ma, Y. Wu, L. Cheng, and D. Yu, "DOA estimation for echo signals and experimental results in the AM radiobased passive radar," IEEE Access, vol. 6, pp. 73316-73327, 2018.

[10] S. J. Davey, G. A. Fabrizio, and M. G. Rutten, "Detection and tracking of multipath targets in over-the-horizon radar," IEEE Transactions on Aerospace and Electronic Systems, vol. 55, no. 5, pp. 2277-2295, 2019.

[11] Z. Zhao, X. Wan, D. Zhang, and F. Cheng, "An experimental study of HF passive bistatic radar via hybrid sky-surface wave mode," IEEE Transactions on Antennas and Propagation, vol. 61, no. 1, pp. 415-424, 2013.

[12] S. Liu, Y. Ma, and Y. Huang, "Sea clutter cancellation for passive radar sensor exploiting multi-channel adaptive filters," IEEE Sensors Journal, vol. 19, no. 3, pp. 982-995, 2019.

[13] P. F. Howland, "Target tracking using television-based bistatic radar," IEE Proceedings - Radar, Sonar and Navigation, vol. 146, no. 3, pp. 166-174, 1999.

[14] Howland and P. Edward, "Television based bistatic radar," Ph.D. dissertation, LAP Lambert Academic Publishing, Sunnyvale, CA, USA, 1997.

[15] B. D. Carlson, E. D. Evans, and S. L. Wilson, "Search radar detection and track with the Hough transform. I. system concept," IEEE Transactions on Aerospace and Electronic Systems, vol. 30, no. 1, pp. 102-108, 1994.

[16] L. R. Moyer, J. Spak, and P. Lamanna, “A multi-dimensional Hough transform-based track-before-detect technique for detecting weak targets in strong clutter backgrounds," IEEE Transactions on Aerospace and Electronic Systems, vol. 47, no. 4, pp. 3062-3068, 2011.

[17] B. Sobhani, T. Zwick, and M. Chiani, "Target TOA association with the Hough transform in UWB radars," IEEE Transactions on Aerospace and Electronic Systems, vol. 52, no. 2, pp. 743754, 2016.

[18] I. S. Reed, R. M. Gagliardi, and M. H. Shao, "Application of three-dimensional filtering to moving target detection," IEEE Transactions on Aerospace and Electronic Systems, vol. AES19, no. 6, pp. 898-905, 1983.

[19] M. Dragovic, Velocity Filtering for Target Detection and Track Initiation, Defence Science and Technology Organisation Salisbury (Australia) Systems Sciences Lab, Adelaide, South Australia, 2003.

[20] H. L. Kennedy, "Efficient velocity filter implementations for dim target detection," IEEE Transactions on Aerospace and Electronic Systems, vol. 47, no. 4, pp. 2991-2999, 2011.

[21] G. Zhou, L. Wang, and T. Kirubarajan, "A pseudo-spectrum approach for weak target detection and tracking," IEEE Transactions on Aerospace and Electronic Systems, vol. 55, no. 6, pp. 3394-3412, 2019.

[22] B. Ristic, S. Arulampalam, and N. Gordon, Beyond the Kalman Filter-Particle Filters for Tracking Applications, Artech House, Norwood, MA, USA, 2004.

[23] W. Yi, L. Fu, Á. F. García-Fernández, L. Xu, and L. Kong, "Particle filtering based track-before-detect method for passive array sonar systems," Signal Processing, vol. 165, pp. 303-314, 2019.

[24] L. Ubeda-Medina, A. F. Garcia-Fernandez, and J. Grajal, "Adaptive auxiliary particle filter for track-before-detect with multiple targets," IEEE Transactions on Aerospace and Electronic Systems, vol. 53, no. 5, pp. 2317-2330, 2017.

[25] F. Lehmann, "Recursive Bayesian filtering for multitarget track-before-detect in passive radars," IEEE Transactions on Aerospace and Electronic Systems, vol. 48, no. 3, pp. 24582480, 2012.
[26] Y. Barniv, "Dynamic programming solution for detecting dim moving targets," IEEE Transactions on Aerospace and Electronic Systems, vol. AES-21, no. 1, pp. 144-156, 1985.

[27] S. M. Tonissen and R. J. Evans, "Peformance of dynamic programming techniques for track-before-detect," IEEE Transactions on Aerospace and Electronic Systems, vol. 32, no. 4, pp. 1440-1451, 1996.

[28] Daikun, S. Zheng, Q. Wang, and Meng, "Dynamic programming track-before-detect algorithm for radar target detection based on polynomial time series prediction," IET Radar, Sonar \& Navigation, vol. 10, no. 8, pp. 1327-1336, 2016.

[29] M. Elhoshy, F. Gebali, and T. A. Gulliver, "Expanding window dynamic-programming-based track-before-detect with order statistics in Weibull distributed clutter," IEEE Transactions on Aerospace and Electronic Systems, vol. 56, no. 4, pp. 25642575, 2020.

[30] X. Tian, J. Liu, M. Mallick, and K. Huang, "Simultaneous detection and tracking of moving-target shadows in ViSAR imagery," IEEE Transactions on Geoscience and Remote Sensing, vol. 99, pp. 1-18, 2020.

[31] H. Wang, J. Yi, and X. Wan, "Greedy algorithm-based trackbefore-detect in radar systems," IEEE Sensors Journal, vol. 18, no. 17, pp. 7158-7165, 2018.

[32] C. F. Olson, "Constrained hough transforms for curve detection," Computer Vision and Image Understanding, vol. 73, no. 3, pp. 329-345, 1999.

[33] D. Yu, H. Ma, L. Cheng et al., "Method and field experiment of target tracking via multi-static Doppler shifts in high-frequency passive radar," IET Radar, Sonar \& Navigation, vol. 10, no. 7, pp. 1201-1212, 2016.

[34] R. Schmidt, "Multiple emitter location and signal parameter estimation," IEEE Transactions on Antennas and Propagation, vol. 34, no. 3, pp. 276-280, 1986.

[35] Y. Li, H. Ma, and L. Cheng, "Iterative robust adaptive beamforming," EURASIP Journal on Applied Signal Processing, vol. 58, no. 1, pp. 1-12, 2017.

[36] J. L. Garry, C. J. Baker, and G. E. Smith, "Evaluation of direct signal suppression for passive radar," IEEE Transactions on Geoscience and Remote Sensing, vol. 99, pp. 1-14, 2017.

[37] H. K. Brustad, Direct Signal Cancellation in Passive Bistatic DVB-T Based Radar, Norwegian Defense Research Establishment (FFI), Lillestrøm, Norway, 2014.

[38] Y. Feng, R. Tao, S. Liu, M. G. Amin, T. Shan, and Y. D. Zhang, "Efficient architecture and hardware implementation of coherent integration processor for digital video broadcast-based passive bistatic radar," IET Radar, Sonar \& Navigation, vol. 10, no. 1, pp. 97-106, 2015.

[39] J. Ji, G. Chen, and L. Sun, “A novel hough transform method for line detection by enhancing accumulator array," Pattern Recognition Letters, vol. 32, no. 11, pp. 1503-1510, 2011.

[40] J. Illingworth and J. Kittler, "A survey of the hough transform," Computer Vision, Graphics, and Image Processing, vol. 44, no. 1, pp. 87-116, 1988.

[41] S. M. Kay, Fundamentals of Statistical Signal Processing. Volume II: Detection Theory, Prentice-Hall, Hoboken, NJ, USA, 1998. 